\title{
Timelike structure functions and hadron multiplicities
}

\author{
Paolo Bolzoni* \\ II. Institut für Theoretische Physik, Universität Hamburg, \\ Luruper Chaussee 149, 22761 Hamburg, Germany \\ E-mail: Paolo.Bolzoni@desy.de
}

In this talk we discuss the results obtained in the new approach that we recently introduced to consider and include both the perturbative and nonperturbative contributions to the evolution of the gluon and quark avarage multiplicities. We report on our progresses in solving a longstanding puzzle of QCD. The new formalism is motivated by recent important theoretical developments in timelike small-x resummation which are also discussed mostly from an historical point of view. We have extended our global analysis to fit the available data adding the strong coupling constant as a fit parameter. In this way our best fit gives $\alpha_{S}\left(M_{z}\right)=0.124 \pm 0.005$ and for the corresponding $\chi^{2}$ we have obtained a further improvement.

XXI International Baldin Seminar on High Energy Physics Problems, September 10-15, 2012

JINR, Dubna, Russia

* Speaker. 


\section{Introduction}

When jets are produced at colliders, they can be initiated either by a gluon or by a quark. Because of the fact that these two partons carry different color charges and spin, it is expected that the corresponding jets show different properties: typically a gluon jet is braoder and contains a larger amount of hadrons. Jets with different parent partons can also be distinguished and investigated looking for the jet charge distribution as discussed in detail in Ref.[1] with important applications for the LHC. The interactions between quarks and gluons are described by quantum chromodynamics (QCD) and to understand the difference between a quark and a gluon jet is one of the strongest test of this theory.

The typical way to depict the production of a jet from a parton is the following: an initial quark (or gluon) start radiating gluons, which in turn can radiate further gluons or split into secondary quark-antiquark pairs. In this way the virtuality of the parent parton decreases in a so called parton showering process. Finally when the virtuality falls below a certain cutoff the cascade stops and the final state partons hadronize into color neutral hadrons, a process usually described by phenomenological models. This happens because the production of hadrons is a typical process where non-perturbative phenomena are involved. However, for particular observables, this problem can be avoided. In particular the "counting" of hadrons in a jet which is initiated at a certain scale $Q^{2}$ belongs to that class of observables and in this case one can assume with quite high accuracy the hypothesis of Local Parton-Hadron Duality (LPHD) which simply states that parton distributions are just renormalized in the hadronization process without changing their shape [2]. This would in principle allow perturbative QCD at fixed order to make predictions without the need to consider phenomenological models of hadronization. Nevertheless these are processes dominated by soft gluon emissions and it is a well known fact that in such kinematic regions of the phase space fixed order perturbation theory fails and resummation is needed (see e.g. Ref.[3]) and this is the main topic discussed in this talk.

The gluon and quark multiplicities $\left\langle n_{h}\left(Q^{2}\right)\right\rangle_{g}$ and $\left\langle n_{h}\left(Q^{2}\right)\right\rangle_{s}$ represent the avarage number of hadrons in a jet initiated by a gluon or a quark respectively at scale $Q^{2}$. In the past analytic predictions have been achieved solving the equations for the generating functionals in the modified leading logarithmic approximation (MLLA) in Ref.[4] up to the so called $\mathrm{N}^{3} \mathrm{LOr}$ in the expansion parameter $\sqrt{\alpha_{s}}$ i.e. $\alpha_{s}^{3 / 2}$. However for the ratio $r=\left\langle n_{h}\right\rangle_{g} /\left\langle n_{h}\right\rangle_{s}$ the theoretical prediction is about $10 \%$ higher than the data at the scale of the $Z^{0}$ vector boson and the difference with the data becomes even larger at lower scales even if the convergence of the perturbative series behaves very well. An alternative approach was given in Ref. [5] where equations for the derivative of the ratio of the multiplicities are obtained in the MLLA within the framework of the colour dipole model. There a constant of integration which is supposed to encode non-perturbative contributions is fixed by the data. A constant offset to the quark and gluon multiplicities has also been introduced in [6].

Very recently a new formalism has been proposed and developed in Refs.[7, 8], where the problem of the apparent good convergence of the perturbative series is solved and where any ad hoc offset is needed once one includes the effects coming from the full mixing between quarks and gluon evolutions. The result looks like a generalization of the result obtained in Ref.[4]. In the new approach the non-perturbative physics to the gluon-quark multiplicities is parametrized simply in the initial conditions of the evolution equations. Due to the good agreement with the 
data obtained in Ref.[8], at the time of the contribution to this conference we have done a study where we have extended our analysis adding as a fit parameter also the strong coupling constant. Thanks to the new outstanding results in small-x timelike resummation obtained in [9] in the $\overline{M S}$ scheme, we were able to compute exact next-to-next-to-leading-logarithm (NNLL) contributions to the evolution of the multiplicity with with approximated NNNLO normalization factors in the $\sqrt{\alpha_{s}}$ expansion. Previously published results where available up to the NLL accuracy but in a massive gluon scheme which in general is unfortunately not suitable to combine resummation with fixed higher order corrections which are naturally given in the $\overline{M S}$ scheme. See Refs.[10, 11] for a general discussion about the scheme choice and scheme dependence in this context.

In the following after reviewing the history which lead to the latest important improvements in "timelike" QCD of Refs.[12,9] and sketching our formalism, we present, as anticipated, the result of a slightly different global fit than in [8] where also the strong coupling contstant has been added among the free parameters confirming the good quality of the fit.

\section{Exiting times for "timelike" QCD}

The important and fundamental results obtained in Refs.[12,9] represent the beginning of the happy end of a quite long story: In the 1972 it was realized in Ref.[13] that at the lowest order the "timelike" splitting functions (occuring in Semi-Inclusive electron positron Annihilation (SIA)) are the same as their "spacelike" counterparts (occuring in Deep Inelastic Scattering (DIS)). This property goes under the name of Gribov-Lipatov relation. Then in the 1980 it has been shown by Curci, Furmanski and Petronzio [14] that the Gribov-Lipatov relation is violated. In that period several groups obtained the NLO contributions to the 'timelike' splitting functions [14, 15, 16, 17, 18, 19]. In 2004 the NNLO "spacelike" splitting functions were published by Moch, Vermaseren and Vogt in Ref.[20, 21]. In this last case the calculation could be performed via forward scattering amplitudes, a fact that has allowed a direct calculation in terms of Feynman diagrams. This is however not the case for the "timelike" splitting functions and different techniques have been investigated. Two of them have been the most successfull. The first one is due to Dokshitzer, Marchesini and Salam [22] who developed a formalism trying to rescue (at least at the formal level) the GribovLipatov relation at higher orders. The approach of Ref.[22] shaded light on many theoretical aspects revealing (using their words) "intrinsic beauty of the perturbative quark-gluon dynamics"[23]. The second one is based on the fact that the "timelike" and the "spacelike" splitting functions can be related by the analytic continuation of the scaling variable $x \rightarrow 1 / x$ with $x$ representing the fraction of the parton longitudinal momentum. In particular, even if beyond the LO this cannot be done directly from the splitting functions, it can be done for the corresponding physical evolution kernels and this has been shown in Refs. [24, 25]. Formally, the evolution of a structure function is governed by a physical kernel $K$ which depends on the coefficient function $C$ and the splitting function $P$ according to:

$$
Q^{2} \frac{\partial F}{\partial Q^{2}}=K \otimes F=\left[\left(\beta\left(\alpha_{s}\right) \frac{d C}{d \alpha_{s}}+C \otimes P\right) \otimes C^{-1}\right] \otimes F,
$$

where $\beta$ is the QCD beta function for the running of the strong coupling $\alpha_{s}$

$$
\beta\left(\alpha_{s}\right)=Q^{2} \frac{\partial \alpha_{s}\left(Q^{2}\right)}{\partial Q^{2}},
$$


and where $\otimes$ means the usual integral convolution with respect to $x$. Eq.(2.1) also tells us that

$$
F=C \otimes D,
$$

where $D$ is what we call fragmentation function (the analog of the parton densities in the "spacelike" case) and that

$$
Q^{2} \frac{\partial D}{\partial Q^{2}}=P \otimes D
$$

According to Eq.(2.1) and the discussion above it, we see that in principle all the "timelike" splitting functions can be obtained from the "timelike" physical kernels (obtained by the analytic continuation $x \rightarrow 1 / x$ ), once the "timelike" coefficient functions $C$ are known. The "timelike" coefficients functions are known at NNLO [26, 27, 28, 29, 30, 31, 32], while the analytic continuation of the physical kernels from the NNLO "spacelike" ones has been obtained in Refs. [33, 34] and finally in Ref.[12]. Here we mention only that the analytic continuation $x \rightarrow 1 / x$ becomes subdle (see Ref.[25]) for the logarithmic terms singular in $x \rightarrow 1$ for which

$$
\ln (1-x) \rightarrow \ln (1-x)-\ln x+i \pi
$$

and that additional constaints (see Ref.[12] and referencies therein) from the momentum sum rules, the supersymmetric limit $\left(C_{A}=C_{F}=n_{f}\right)$ and from the relations found in Ref.[22] are needed to close the problem in a satisfactory way.

Nevertheless the NNLO "timelike" splitting functions for the quark-singlet system obtained in Ref.[12] are not the end of the story. Indeed they present singularities in the threshold $(x \rightarrow$ $1)$ and in the large energy limit $(x \rightarrow 0)$. Both kind of singularities are due to the radiation of soft gluons and they make perturbation theory to fail. As already mentioned, the difficulty with these singularities are overcome by resummation. For very recent developments in the large- $\mathrm{x}$ resummation see e.g. Refs.[35, 36]. Here we are mainly interested in the small-x resummation because it is directly related to the computation of hadronic multiplicities as already realized a long time ago [3]. With respect to the multiplicity studies, the basic equation is the one governing the evolution of fragmentation function $D$ for the gluon-singlet system Eq.(2.4), which in Mellin space sees the convolutional product $\otimes$ turn into an ordinary matrix product:

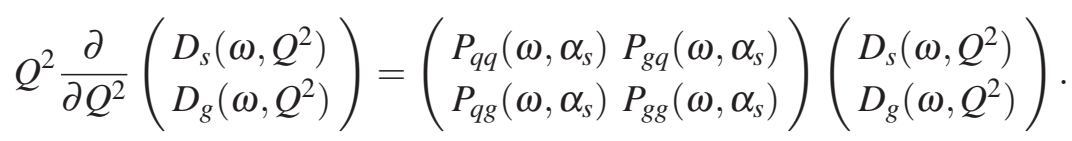

Here $\omega=N-1$ with $N$ the standard Mellin moments with respect to $x$ and where $P_{i j}$ are splitting functions as introduced in Eq.(2.1). The standard definition for the hadron multiplicity in terms of the fragmentation function is given by (see e.g. Ref.[37]) the integral over $x$ of the fragmentation function which is just the first Mellin moment $(\omega=0)$ :

$$
\left\langle n_{h}\left(Q^{2}\right)\right\rangle_{a} \equiv\left[\int_{o}^{1} d x x^{\omega} D_{a}\left(x, Q^{2}\right)\right]_{\omega=0}=D_{a}\left(\omega=0, Q^{2}\right),
$$

where $a=s$ for a quark jet and $a=g$ for a gluon jet. It is clear from the definition in Eq.(2.7) that eventual non-integrable singularities in $x \sim 0$ in the inegrand could not occur for the definition to make sense. We remind that the singularities in $x=0$ are translated into singularities in $\omega=$ 
0 . We have already mentioned that resummation which includes the singularities from all orders according to a certain logarithmic accuracy is the standard way to solve this problem. Indeed after resummation, the singular behavior in $x \sim 0$ (or equivalently in $\omega \sim 0$ ) disappears. The generally better choice of the $\overline{M S}$ scheme for to perform resummation has been considered and solved only quite recently. Firstly the NLL accuracy has been obtained in Refs.[38, 39] and finally the NNLL accuracy has been reached in Ref.[9]. Here thanks to the approach used by A. Vogt in Ref.[38], the singularities of the splitting functions are iteratively extracted according to the all order factorization into the transition function $Z(\varepsilon)$ of the $\varepsilon=0$ poles in dimensional regularization $(d=4-2 \varepsilon)$. Indeed, according to the factorization theorem [40, 14], we can rewrite Eq.2.3 in Mellin space as

$$
F=C D=C^{0}(\varepsilon) Z^{-1}(\varepsilon) Z(\varepsilon) D^{0},
$$

where $C^{0}$ and $D^{0}$ are the "bare" coefficient function and fragmentation function. $Z(\varepsilon)$ is the transition function containing only poles in $\varepsilon=0$ that are factored out from $C^{0}$. Hence, substituting Eq.(2.8) into Eq.(2.4) we get that the splitting functions can be directly related to the transition function in the following way:

$$
P=Q^{2} \frac{\partial Z}{\partial Q^{2}} Z^{-1}=\beta\left(\alpha_{s}\right) \frac{\partial Z}{\partial \alpha_{s}} Z^{-1}
$$

Now it is shown in Ref.[38] how one can solve this equation in $Z$ obtaining at all orders the three highest order poles in $\varepsilon$ knowing the NNLO corrections to $P$ and $\beta$. Additionally knowing the higher order corrections to $C$ which is pole free one obtains from the NNLO computations the all order structure of the three first highest singularities in $\varepsilon$ :

$$
C^{0}(\varepsilon)=C(\varepsilon) Z(\varepsilon)
$$

The key point of Ref.[38] is that they realized that for example for the case of the gluon the small $\omega$ behavior of the bare coefficient function is

$$
C^{0}(\varepsilon)=\frac{1}{\omega} \sum_{n} \frac{\alpha_{s}^{n}}{\varepsilon^{2 n-1}} \sum_{l=0}^{n-1} \frac{1}{1-2(n-l) \varepsilon / \omega}\left(A_{l n}+\varepsilon B_{l n}+\varepsilon^{2} C_{l n}+\ldots\right) .
$$

Finally comparing Eq.(2.11) with Eq.(2.10) one obtaines sytems of equations for the coefficients $A, B$ and $C$, which produce sequences up to arbitrary orders in $\alpha_{s}$ of the three highest powers in $1 / \omega$ or equivalently (back to $x$-space) in $\ln x$. Then the highly non trivial part of this approach is the solution of these sequences that are obtained for the large logarithms and this has been successfully obtained in Refs.[38, 9].

\section{Plus-minus component evolution for the gluon-singlet system}

In Ref.[8] it has been shown that, by use of the results obtained in Ref.[9], the scale dependence on $Q^{2}$ of the gluon and quark multiplicities defined by Eq.(2.7) and governed by Eq.(2.6) can be computed analytically in a closed form. The approach that we used is a generalization of the techniques used in Ref.[41] used to solve the evolution equation of the parton densities in the 
"spacelike" case. Our result can be written in the following way

$$
\begin{aligned}
\left\langle n_{h}\left(Q^{2}\right)\right\rangle_{g} & =\left\langle n_{h}\left(Q_{0}^{2}\right)\right\rangle_{g} \hat{T}_{+}\left(Q^{2}, Q_{0}^{2}\right), \\
\left\langle n_{h}\left(Q^{2}\right)\right\rangle_{s} & =\left\langle n_{h}\left(Q_{0}^{2}\right)\right\rangle_{g} \frac{\hat{T}_{+}\left(Q^{2}, Q_{0}^{2}\right)}{r_{+}\left(Q^{2}\right)}+\left[\left\langle n_{h}\left(Q_{0}^{2}\right)\right\rangle_{s}-\frac{\left\langle n_{h}\left(Q_{0}^{2}\right)\right\rangle_{g}}{r_{+}\left(Q_{0}^{2}\right)}\right] \hat{T}_{-}\left(Q^{2}, Q_{0}^{2}\right),
\end{aligned}
$$

where $Q_{0}^{2}$ is an arbitrary reference scale (see below). The dependence on $Q^{2}$ is always inside the running coupling constant $\alpha_{s}\left(Q^{2}\right)$. Indeed substituting the QCD values for the color factors and choosing $n_{f}=5$ in the formulae given in Ref.[8] we can write that at NNLL

$$
\begin{aligned}
\hat{T}_{-}\left(Q^{2}, Q_{0}^{2}\right)= & {\left[\frac{\alpha_{s}\left(Q^{2}\right)}{\alpha_{s}\left(Q_{0}^{2}\right)}\right]^{d_{1}} } \\
\hat{T}_{+}\left(Q^{2}, Q_{0}^{2}\right)= & \exp \left\{d_{2}\left(\frac{1}{\sqrt{\alpha_{s}\left(Q^{2}\right)}}-\frac{1}{\sqrt{\alpha_{s}\left(Q_{0}^{2}\right)}}\right)+d_{3}\left(\sqrt{\alpha_{s}\left(Q^{2}\right)}-\sqrt{\alpha_{s}\left(Q_{0}^{2}\right)}\right)\right\} \\
& \times\left[\frac{\alpha_{s}\left(Q^{2}\right)}{\alpha_{s}\left(Q_{0}^{2}\right)}\right]^{d_{4}},
\end{aligned}
$$

where

$$
d_{1}=0.38647, \quad d_{2}=2.65187, \quad d_{3}=-3.87674, \quad d_{4}=0.97771 .
$$

The function $r_{+}\left(Q^{2}\right)$ in Eq.(3.1) has been computed in [42, 4, 43] up to the third order (NNNLO) in the $\sqrt{\alpha_{s}}$ expansion and in QCD is given by:

$$
r_{+}\left(Q^{2}\right)=2.25-0.61567 \sqrt{\alpha_{s}\left(Q^{2}\right)}-2.19156 \alpha_{s}\left(Q^{2}\right)+0.24348 \alpha_{s}^{3 / 2}\left(Q^{2}\right)+O\left(\alpha_{s}^{2}\right),
$$

again with $n_{f}=5$. The first term in Eq.(3.4) corresponds to the lowest order expectation for the ratio between the gluon and the quark multiplicities given by $C_{A} / C_{F}$. According to Eq.(3.1), our prediction for the multiplicity ratio $r$ is

$$
r\left(Q^{2}\right)=\frac{r_{+}\left(Q^{2}\right)}{1+\frac{r_{+}\left(Q^{2}\right)}{r_{+}\left(Q_{0}^{2}\right)}\left(\frac{\left\langle n_{h}\left(Q_{0}^{2}\right)\right\rangle_{s} r_{+}\left(Q_{0}^{2}\right)}{\left\langle n_{h}\left(Q_{0}^{2}\right)\right\rangle_{g}}-1\right) \frac{\hat{T}_{-}\left(Q^{2}, Q_{0}^{2}\right)}{\hat{T}_{+}\left(Q^{2}, Q_{0}^{2}\right)}} .
$$

From this expression we can see that our result is a generalization of the result in Eq.(3.4) due to the inclusion of the contribution proportional to $\hat{T}_{-}\left(Q^{2}, Q_{0}^{2}\right)$ in the denominator of Eq.(3.5).

Now we show that our results in Eqs.(3.1,3.5) do not depend on the reference scale $Q_{0}^{2}$ and doing this also the meaning of $\hat{T}_{ \pm}\left(Q^{2}, Q_{0}^{2}\right)$ will become clear. We define the so called "plus" and "minus" component in terms of the singlet and the gluon components defined in Eq.(2.7) in the following way:

$$
\begin{aligned}
& \left\langle n_{h}\left(Q^{2}\right)\right\rangle_{+} \equiv\left\langle n_{h}\left(Q^{2}\right)\right\rangle_{g}, \\
& \left\langle n_{h}\left(Q^{2}\right)\right\rangle_{-} \equiv\left\langle n_{h}\left(Q^{2}\right)\right\rangle_{s}-\frac{\left\langle n_{h}\left(Q^{2}\right)\right\rangle_{g}}{r_{+}\left(Q^{2}\right)} .
\end{aligned}
$$


With this definition we have that the system of Eqs.(3.1) is equivalent to the following one

$$
\left\langle n_{h}\left(Q^{2}\right)\right\rangle_{ \pm}=\left\langle n_{h}\left(Q_{0}^{2}\right)\right\rangle_{ \pm} \hat{T}_{ \pm}\left(Q^{2}, Q_{0}^{2}\right)
$$

By noting that according to Eqs.(3.2),

$$
\hat{T}_{ \pm}\left(Q^{2}, Q_{0}^{2}\right) \hat{T}_{ \pm}\left(Q_{0}^{2}, Q_{1}^{2}\right)=\hat{T}_{ \pm}\left(Q^{2}, Q_{1}^{2}\right)
$$

one can directly check that, after expressing $\left\langle n_{h}\left(Q_{0}^{2}\right)\right\rangle_{ \pm}$in terms of $Q_{1}^{2}$ using Eqs.(3.7) and then replacing them again into Eqs.(3.7), one arrives at the same expression but with $Q_{0}^{2}$ replaced by $Q_{1}^{2}$. This shows the independence of the result on the reference scale $Q_{0}^{2}$ which is arbitrary and that $\hat{T}_{ \pm}\left(Q^{2}, Q_{0}^{2}\right)$ just represent the renormalization group exponents in a basis where the splitting function matrix of Eq.(2.6) can be considered diagonal. To look for a basis where the splitting function matrix could be considered diagonal by a sufficient accuracy was the main motivation under the computation in Ref.[8].

\section{Global fit to the avarage multiplicity data: determination of $\alpha_{s}$}

Our result Eqs.(3.1) depends on three quantities that should be fixed by the comparison with the experiment. They are $\left\langle n_{h}\left(Q_{0}^{2}\right)\right\rangle_{g},\left\langle n_{h}\left(Q_{0}^{2}\right)\right\rangle_{s}$ (representing the avarage multiplicity at the reference scale $Q_{0}^{2}$ ) and a reference value for the running coupling constant which is conventionally chosen at the scale of the mass of the $Z^{0}$ weak boson, $\alpha_{s}\left(M_{Z}\right)$. In Ref.[8], $\alpha_{s}\left(M_{Z}\right)$ has been fixed as an input parameter to be equal to the world avarage value [44]. The result that we obtained was

$$
\begin{aligned}
\left\langle n_{h}\left(Q_{0}^{2}\right)\right\rangle_{g} & =24.02 \pm 0.36, \quad\left\langle n_{h}\left(Q_{0}^{2}\right)\right\rangle_{g}=15.83 \pm 0.37, \quad 90 \% \text { C.L. } \\
\alpha_{s}\left(M_{Z}\right) & =0.118, \quad \text { input parameter }
\end{aligned}
$$

where the arbitrary scale has been chosen to be $Q_{0}=50 \mathrm{GeV}$. We have checked that the $\chi^{2}$ remains unchanged changing the value of $Q_{0}^{2}$ as expected from the considerations done above.

Due to the quite good agreement with the data obtained in Ref.[8], we tried to treat $\alpha_{s}\left(M_{Z}\right)$ as an additional fit parameter. The result that we obtained for such a fit (always for $Q_{0}=50 \mathrm{GeV}$ ) is:

$$
\begin{aligned}
\left\langle n_{h}\left(Q_{0}^{2}\right)\right\rangle_{g} & =24.18 \pm 0.32, \quad\left\langle n_{h}\left(Q_{0}^{2}\right)\right\rangle_{g}=15.86 \pm 0.37, \quad 90 \% \text { C.L. } \\
\alpha_{s}\left(M_{Z}\right) & =0.124 \pm 0.005, \quad 90 \% \text { C.L. }
\end{aligned}
$$

which is in agreement with Eq.(4.1) and with the experimental values for the gluon and quark multiplicities at $50 \mathrm{GeV}$. The $\chi^{2}$ per degree of freedom is improved in this fit from 3.71 (corresponding to Eqs.(4.1)) down to 2.84 (corresponding to Eqs.(4.2)). The predicted value for $\alpha_{s}\left(M_{Z}\right)$ is higher than the world avarage value [44], however such a higher value is not new in hadronic final states of electron-positron annihilation [45]. However how much it would make sense to compare our result with $\alpha_{s}^{\overline{M S}}$ is still under investigation. For the determination of the result in Eqs.4.2 we have used the same data selection we used in Ref.[8], which is also the one adopted by the DELPHI colaboration in Ref.[46]. In Fig.1 we plot the result of our new fir according to Eq.(4.2) together with the uncertainties and the data, which are taken from the Tables in Ref.[47]. 


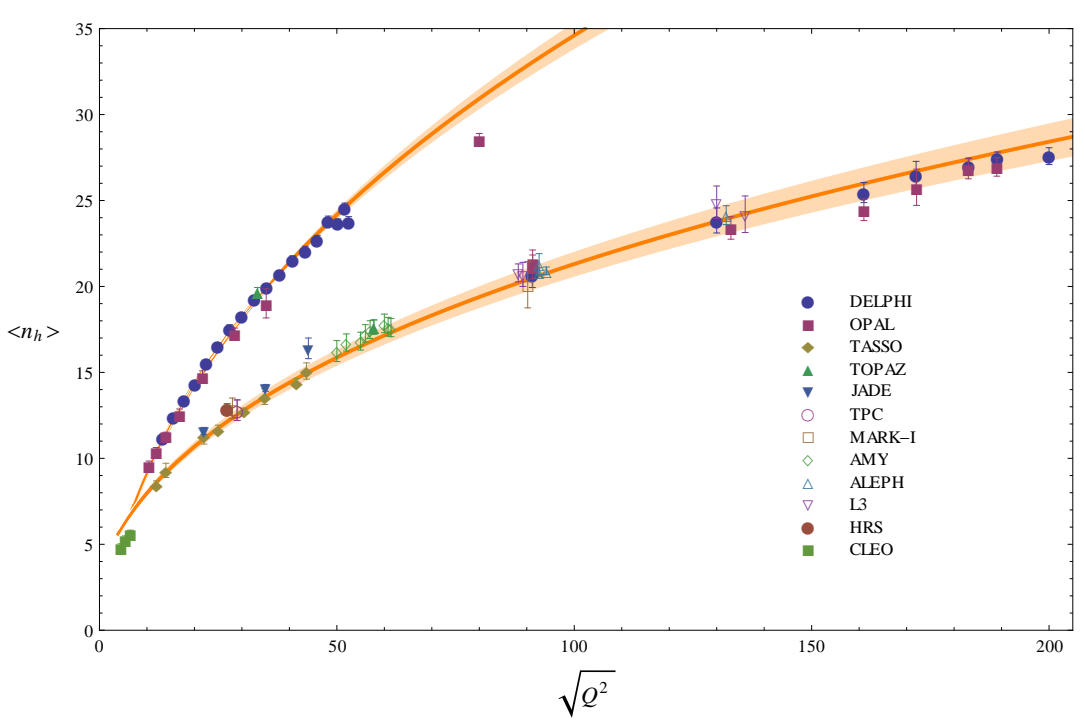

Figure 1: Gluon and quark multiplicities fits according to Eq.(4.2) compared to the data.

\section{Conclusions}

In this talk we have sketched the history and the various techniques culminating in the very important new results from the theoretical side (see Refs.[12,9] and referencies therein for the most recent developments), which have already made possible new important improvement in the descriprion of the data in hadronic final states processes in the "timelike" case (see Ref.[8]). Motivited by the well description of the data obtained in Ref.[8] for the avarage multiplicities in jets initiated by gluon and quarks, we have extended our previous analysis making the value of the strong coupling constant at the reference scale of the mass of the $Z^{0}$ boson a parameter for the fit. In this way we have still obtained numbers in agreement with observations for the value of the multiplicities at the reference scale $Q_{0}=50 \mathrm{GeV}$ and in addition we got a significant improvement in the $\chi^{2}$ per degree of freedom and, as a byproduct, a prediction for $\alpha_{s}\left(M_{Z}\right)$.

Acknowledgements: I would like to thank the organizers of the "XXI International Baldin Seminar on High Energy Physics Problems" and A. Kotikov for fruitful discussions and for inviting me to this conference. This work is in part supported by the Heisenberg-Landau program and in part by the German Federal Ministry for Education and Research BMBF through Grant No. 05 HT6GUA, by the German Research Foundation DFG through the Collaborative Research Centre No. 676 Particles, Strings and the Early Universe-The Structure of Matter and Space Time, and by the Helmholtz Association HGF through the Helmholtz Alliance Ha 101 Physics at the Terascale.

\section{References}

[1] W.J. Waalewijn, (2012), arXiv:1209.3019.

[2] Y.I. Azimov et al., Z. Phys. C27 (1985) 65.

[3] A.H. Mueller, Phys. Lett. B104 (1981) 161. 
[4] A. Capella et al., Phys. Rev. D61 (2000) 074009, [hep-ph/9910226].

[5] P. Eden and G. Gustafson, JHEP 09 (1998) 015, [hep-ph/9805228].

[6] DELPHI Collaboration, P. Abreu et al., Phys.Lett. B449 (1999) 383, [hep-ex/9903073].

[7] P. Bolzoni, (2012), arXiv:1206.3039.

[8] P. Bolzoni, B. Kniehl and A. Kotikov, (2012), arXiv:1209.5914.

[9] C.H. Kom, A. Vogt and K. Yeats, JHEP 1210 (2012) 033, [arXiv:1207.5631].

[10] S. Albino et al., (2011), arXiv:1107.1142.

[11] S. Albino et al., Nucl. Phys. B851 (2011) 86, [arXiv:1104.3018].

[12] A. Almasy, S. Moch and A. Vogt, Nucl.Phys. B854 (2012) 133, [arXiv:1107.2263].

[13] V.N. Gribov and L.N. Lipatov, Sov. J. Nucl. Phys. 15 (1972) 438.

[14] G. Curci, W. Furmanski and R. Petronzio, Nucl. Phys. B175 (1980) 27.

[15] W. Furmanski and R. Petronzio, Phys. Lett. B97 (1980) 437.

[16] J. Kalinowski et al., Nucl. Phys. B181 (1981) 253.

[17] J. Kalinowski, K. Konishi and T. Taylor, Nucl.Phys. B181 (1981) 221.

[18] E. Floratos, C. Kounnas and R. Lacaze, Nucl.Phys. B192 (1981) 417.

[19] T. Munehisa et al., Prog.Theor.Phys. 67 (1982) 609.

[20] S. Moch, J. Vermaseren and A. Vogt, Nucl.Phys. B688 (2004) 101, [hep-ph/0403192].

[21] A. Vogt, S. Moch and J. Vermaseren, Nucl.Phys. B691 (2004) 129, [hep-ph/0404111].

[22] Y. Dokshitzer, G. Marchesini and G. Salam, Phys.Lett. B634 (2006) 504, [hep-ph/0511302].

[23] Y. Dokshitzer, Phys.Atom.Nucl. 71 (2008) 636.

[24] J. Blumlein, V. Ravindran and W. van Neerven, Nucl.Phys. B586 (2000) 349, [hep-ph/0004172].

[25] M. Stratmann and W. Vogelsang, Nucl.Phys. B496 (1997) 41, [hep-ph/9612250].

[26] P.J. Rijken and W.L. van Neerven, Phys. Lett. B392 (1997) 207, [hep-ph/9609379].

[27] E. Zijlstra and W. van Neerven, Phys.Lett. B273 (1991) 476.

[28] W. van Neerven and E. Zijlstra, Phys.Lett. B272 (1991) 127.

[29] P.J. Rijken and W.L. van Neerven, Nucl. Phys. B487 (1997) 233, [hep-ph/9609377].

[30] S. Moch and J. Vermaseren, Nucl.Phys. B573 (2000) 853, [hep-ph/9912355].

[31] J. Blumlein and V. Ravindran, Nucl. Phys. B749 (2006) 1, [hep-ph/0604019].

[32] A. Mitov and S. Moch, Nucl. Phys. B751 (2006) 18, [hep-ph/0604160].

[33] A. Mitov, S. Moch and A. Vogt, Phys. Lett. B638 (2006) 61, [hep-ph/0604053].

[34] S. Moch and A. Vogt, Phys. Lett. B659 (2008) 290, [arXiv:0709.3899].

[35] N. Lo Presti, A. Vogt and A. Almasy, (2012), arXiv:1202.5224.

[36] G. Grunberg, Nucl.Phys. B851 (2011) 30, [arXiv:1101.5377].

[37] R.K. Ellis, W.J. Stirling and B. Webber, Camb.Monogr.Part.Phys.Nucl.Phys.Cosmol. 8 (1996) 1. 
[38] A. Vogt, JHEP 10 (2011) 025, [arXiv:1108.2993].

[39] S. Albino et al., Nucl. Phys. B855 (2012) 801, [arXiv:1108.3948].

[40] R.K. Ellis et al., Nucl. Phys. B152 (1979) 285.

[41] A.J. Buras, Rev. Mod. Phys. 52 (1980) 199.

[42] I. Dremin and J. Gary, Phys.Lett. B459 (1999) 341, [hep-ph/9905477].

[43] I.M. Dremin and J.W. Gary, Phys. Rept. 349 (2001) 301, [hep-ph/0004215].

[44] S. Bethke, (2012), arXiv:1210.0325.

[45] G. Dissertori et al., JHEP 0908 (2009) 036, [arXiv:0906.3436].

[46] DELPHI Collaboration, J. Abdallah et al., Eur.Phys.J. C44 (2005) 311, [hep-ex/0510025].

[47] M. Siebel, (2003), WU-B-DIS-2003-11; PHD thesis Bergische Universität Wuppertal, Germany. 